\title{
Serum complement proteomics reveal biomarkers for hypertension disorder of pregnancy and the potential role of Clusterin
}

Shanshui Zeng ${ }^{1 \dagger}$, Mengru Han ${ }^{1 \dagger}$, Min Jiang ${ }^{1}$, Fei Liu ${ }^{1}$, Yanwei Hu${ }^{1}$, Yan Long ${ }^{1}$, Chunyan Zhu², Fangling Zeng ${ }^{3}$, Qiangsheng $\mathrm{Gan}^{2}$, Weitao $\mathrm{Ye}^{2}$, Wenjin $\mathrm{Fu}^{4^{*}}$ and Hongling Yang ${ }^{1 *}$ (D)

\begin{abstract}
Introduction: Hypertension disorder of pregnancy (HDP) is one of the leading causes of maternal and foetal illness. The aim of the current study was to identify and verify novel serum markers for HDP.

Methods: A label-free LC-MS/MS method was used to establish the serum proteomic profiles of 12 pre-HDP (before clinical diagnosis of HDP) pregnancies and verify prioritized candidates in the verification set of 48 pre-HDP pregnancies. These biomarkers were revalidated by ELISA in an independent cohort of 88 pre-HDP pregnancies. Subsequently, the candidate biomarkers were histologically analysed by immunohistochemistry, and function was evaluated in TEV-1 cells.

Results: We identified 33 proteins with significantly increased abundance and 14 with decreased abundance (peptide FDR $\leq 1 \%, P<0.05$ ). Complement was one of the top enriched components in the pre-HDP group compared with the control group. Three complement factors (CLU, CFHR5, and CRP) were significantly increased in the three sets, of which CLU was a critical factor for the development of HDP $(O R=1.22, P<0.001)$. When these three factors and body weight were combined, the AUC was 0.74 , with a sensitivity of 0.67 and specificity of 0.68 for HDP prediction compared with normal pregnancy. In addition, inflammation-induced CLU could inhibit the invasion of TEV-1 cells.
\end{abstract}

Conclusions: Complement proteins may play an essential role in the occurrence of HDP by acting on trophoblast cells. CLU may be a high-risk factor for HDP, and the models combining candidates show reasonable screening efficiency of HDP in the first half of pregnancy.

Keywords: Hypertensive disorder of pregnancy, Label-free LC-MS/MS, Complement system, Clusterin, Trophoblast

\footnotetext{
*Correspondence: 332689892@qq.com; hlyang62@126.com

†'Shanshui Zeng and Mengru Han have equal contributions and should be considered as co-first authors.

${ }^{4}$ Clinical Laboratory, Houjie Hospital of Guangdong Medical University,

HeTian Road, Dongguan 523945, Guangdong, China

'Department of Laboratory, Guangzhou Women and Children's Medical

Centre, Guangzhou Medical University, No.9, Jinsui Road, Guangzhou 510623, Guangdong, China

Full list of author information is available at the end of the article
}

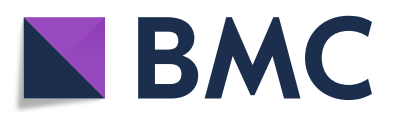

(c) The Author(s). 2021 Open Access This article is licensed under a Creative Commons Attribution 4.0 International License, which permits use, sharing, adaptation, distribution and reproduction in any medium or format, as long as you give appropriate credit to the original author(s) and the source, provide a link to the Creative Commons licence, and indicate if changes were made. The images or other third party material in this article are included in the article's Creative Commons licence, unless indicated otherwise in a credit line to the material. If material is not included in the article's Creative Commons licence and your intended use is not permitted by statutory regulation or exceeds the permitted use, you will need to obtain permission directly from the copyright holder. To view a copy of this licence, visit http://creativecommons.org/licenses/by/4.0/. The Creative Commons Public Domain Dedication waiver (http://creativecommons.org/publicdomain/zero/1.0/) applies to the data made available in this article, unless otherwise stated in a credit line to the data. 


\section{Introduction}

Hypertensive disorder of pregnancy (HDP) is an important cause of severe acute morbidity, long-term disability and mortality among mothers and babies, affecting up to $10 \%$ of pregnancies worldwide [1, 2]. Gestational hypertension $(\mathrm{GH})$ and preeclampsia $(\mathrm{PE})$ are subtypes of HDP that are commonly characterized by hypertension symptoms after 20 weeks of pregnancy. PE is a more severe subtype due to proteinuria and/or liver, kidney, and nervous system damage. Although blood pressure returns to normal after delivery, mothers with HDP and their offspring are still at high risk of cardiovascular disease. HDP is associated with various maternal high-risk factors, such as antiphospholipid antibodies, previous diabetes, multiple (twin) pregnancy, and family history of HDP [3]. The pathogenesis of HDP remains complex and diverse, and no single theory can fully explain its mechanism; thus, there is no effective prediction or treatment choice in clinical practice [4].

The current consensus is that early prediction contributes to prevention, diagnosis, and clinical intervention and can improve the prognosis of HDP. Recent studies have focused on evaluating the response of soluble vascular factor levels to disease activity for early diagnosis. However, their early prediction ability is insufficient. There is still an urgent need to identify other useful biomarkers to help predict HDP $[5,6]$.

An increasing number of studies have highlighted that systemic inflammation and imbalance of the maternal immune system may play a critical role in the pathogenesis of HDP [7]. Our previous results have demonstrated that inflammatory dysfunction may be detected early before the clinical onset of $\mathrm{PE}$ and as early as the first trimester of pregnancy $[8,9]$. A recent study has shown that the overexpression of complement component C5a and C5a-receptor in the placenta can lead to maternal arterial stiffness and increased blood pressure [10]. HDP is associated with an adaptive collapse of tolerogenic cells, including a shift in $\mathrm{T}$ cell distribution towards Th1 and Th17, away from Th2 and regulatory $\mathrm{CD}^{+} \mathrm{T}$ cell (Treg) populations [11]. For this reason, it is of great clinical importance to identify the inflammatory and immune status in maternal blood that can predict and help prevent HDP in the preclinical state. Given the phenotypic relevance and stability of serum proteins under specific conditions, Geyer et al. proposed to use serum as a viable diagnostic biomarker source [12]. Serum contains a wide range of proteins that can act as biological signals for physiological states during homeostasis or perturbation. Recent advances in proteomics enable comprehensive evaluations of low- and high-abundance proteins in various body fluids $[13,14]$.

Clusterin (CLU) is a stress-activated, ATP-independent chaperone molecule that is usually secreted from cells.
CLU is a new pleiotropic factor potentially involved in stimulating inflammatory cytokines such as Interleukin (IL6) and regulation of lipid metabolism, cell differentiation, cell invasion and tissue remodeling $[15,16]$. The occurrence of HDP is related to insufficient trophoblast cell invasion and excessive inflammation. In this context, CLU may involve in the development of HDP.

In the current study, we used a label-free nano LCMS/MS method to characterize serum proteomic profiles of pre-HDP (before 20 weeks of gestation) and healthy pregnant women. We also tried to understand whether these potential markers have predictive significance for HDP when used alone or in combination and to explore the role of CLU in the development of HDP.

\section{Materials and methods Study participants}

This study included participants from two independent cohorts. Participants from Guangzhou Women and Children's Medical Center (Guangzhou, China) were recruited from November 2014 to April 2017. Furthermore, participants from the Maternal \& Child Health Hospital of Foshan (Foshan, China) cohort were recruited from Jul 2017 to Jun 2019. The Ethics Committee of Guangzhou Women and Children's Medical Center and Maternal \& Child Health Hospital of Foshan approved all aspects of this study (Ethics number: 2018030306). Written informed consent was obtained from all subjects. Two experienced obstetricians and gynaecologists assessed HDP and healthy pregnancies in the cohorts.

The definition of HDP by the American College of Obstetricians and Gynaecologists (ACOG) [4] includes $\mathrm{GH}$ (new-onset systolic blood pressure $>140 \mathrm{mmHg}$ or diastolic blood pressure $>90 \mathrm{mmHg}$ without significant proteinuria after 20 weeks of pregnancy) and PE (newonset hypertension with proteinuria $>0.3 \mathrm{~g} /$ day or with other maternal organ dysfunction detailed in the Data supplement). Cases with a history of hypertension, nephropathy, or other diseases leading to elevated blood pressure before the 20th week of pregnancy were excluded. Furthermore, patients with primary abnormal lipid metabolism, multiple pregnancies, miscarriage, stillbirth, foetal malformations, gestational diabetes, pregnancy with thyroid disease, and pregnancy with liver and kidney disease were also excluded.

According to the inclusion and exclusion criteria, women who later developed HDP (pre-HDP) and 1:1matched normal pregnancies (matched for maternal age, gestational age at sample collection, and sample storage time) were enrolled in this study (Fig. S1). The cases from Guangzhou Women and Children's Medical Center were randomly divided into the screening set $(12$ HDP and 12 control cases) and development set (22 pre- 
GH, 26 pre-PE, and 48 control cases) to establish a prediction model. Cases from the Maternal \& Child Health Hospital of Foshan (29 pre-GH, 59 pre-PE, and 88 control cases) were used to validate the model.

\section{Serum and peptide processing}

Fresh venous blood samples $(2 \mathrm{~mL})$ were collected from the participants before the 20th week of gestation and then centrifuged $\left(1200 \times g\right.$ for $10 \mathrm{~min}$ at $\left.18^{\circ} \mathrm{C}\right)$. Peptides were prepared following a previous protocol [13]. Briefly, peptides were stored at $-80^{\circ} \mathrm{C}$ after removal of highabundance proteins, reduction, alkylation, trypsin digestion and removal of ions.

\section{Serum proteomic analysis}

$L C-M S$ analysis. In the two sets, serum $\mathrm{C}$ proteomics was analysed in triplicate using quantification label-free high-pressure liquid chromatography combined with mass spectrometry nano-LC-MS/MS (Thermo Fisher Scientific, Waltham, MA, USA). For each run, $1 \mu \mathrm{g}$ of the digest was injected onto a $100 \mu \mathrm{m}$ i.d. $\times 100 \mathrm{~mm}$ reverse-phase $\mathrm{C} 18 \mathrm{BEH}$ column with $1.7 \mu \mathrm{m}$ particles and a 300 A pore size using a nano Acquity system (Waters, Milford, MA, USA). The chromatographic solvents were water (A) and acetonitrile (B), both with $0.1 \%$ formic acid. Peptides were eluted from the column over a gradient of 3-35\% B (130 min). At $140 \mathrm{~min}$, the gradient was increased to $95 \% \mathrm{~B}$ and held for $10 \mathrm{~min}$. At $160 \mathrm{~min}$, the gradient returned to $3 \%$ to re-equilibrate the column for the next injection. A linear gradient blank was run for 50 min between samples to prevent sample carryover. A quality control sample was tested after every 10th experimental sample. Data-dependent MS/MS-analysed peptides were eluted from the column on label-free nano LC-MS/MS. The top-15 method was used for data collection. Briefly, the instrument was set up as follows: the resolution of the MS scan was set to 70,000, and the resolution of the data-dependent MS/MS scan was set to 17,500 to improve speed. The MS AGC target was set to 106 counts, while the MS/MS AGC target was set to 105. The MS scan range was $300-2000 \mathrm{~m} / \mathrm{z}$. MS scans were recorded in profile mode and MS/MS in centroid mode to reduce the data file size. Dynamic exclusion was set to a repeat count of 1 and a duration of $25 \mathrm{~s}$.

\section{Protein identification}

The raw MS files were processed using MaxQuant (version 1.5.6.0). The human protein sequence database (UniProt_human_2016_09) was downloaded from UNIPROT. This database and its reverse decoy were then searched against using MaxQuant. Trypsin was set as a specific enzyme with up to two missing cleavages. Oxidation (M) and acetyl (protein N-term) were considered variable modifications, and carbamidomethyl $(\mathrm{C})$ was set as the fixed modification. The minimum and maximum peptide lengths were seven and 4600 , respectively. Both the peptide and protein false discovery rates (FDRs) were $<0.01$.

\section{Bioinformatics}

A volcano plot and heatmap were used to evaluate the proteomic profile cluster and functional analysis. Heatmap was set considering $P$-values between the two groups and the Ward's methodology as clustering algorithm. When the fold change (FC) was $>1.2$ or $<$ 0.8 and the $\mathrm{P}$-value was $<0.05$, serum protein was considered the protein of interest. For differentially expressed proteins, functional annotation was performed using the DAVID and Gene Ontology (GO) databases. Metascape software (http://metascape.org/) was used to perform protein-protein interaction (PPI) and pathway clustering [17].

\section{Candidate biomarker evaluation}

Human CLU (Abcam, Cambridge, UK) and complement factor H-related protein 5 (CFHR5, Raybiotech, Norcross, GA, USA) immunoassays were used to determine the levels of CLU and CFHR5 in another group of pregnant women (second validation set) before 8-20 weeks of gestation $(n=88)$. The procedure was performed according to each manufacturer's instructions. Serum Creactive protein (CRP) was detected by an i7600 automatic biochemistry analyser (Hitachi, Japan).

\section{Analysis of prediction efficiency}

First, the performance of the verified candidate proteins was evaluated by calculating the ROC curves of the individual and combined proteins in each verification cohort. ROC analysis was performed by setting diagnostic HDP (after 20 weeks) as a positive test. When performing combination marker analysis, the probability is calculated by logistic regression, and the ROC curve is combined to evaluate the predictive ability of the model. The coordinates of the curve were then output to estimate the potential cut-off value.

\section{Immunohistochemistry}

Placental tissues of women with PE and normal pregnancy were obtained immediately after delivery. Following collection, the tissue was extensively washed in PBS and fixed in $4 \%$ neutral buffered formalin for $24 \mathrm{~h}$. The paraffin-embedded placental sections $(3 \mu \mathrm{m})$ were deparaffinized through xylene and rehydrated by graded ethanol. Antigen retrieval was performed using EDTA antigen retrieval solution ( $\mathrm{pH} 8.0)$ and microwave heating $(15 \mathrm{~min})$. Then, 3\% hydrogen peroxide was used to quench endogenous peroxidase activity, and the slides were blocked with 5\% BSA. Afterward, 
they were stained using anti-CLU antibodies (1:100, Affinity, USA) and incubated overnight at $4{ }^{\circ} \mathrm{C}$. The Dako REAL ${ }^{\text {Tx }}$ EnVision $^{\text {Tx }}$ Detection System, Peroxidase/ $\mathrm{DAB}+$, and Rb/Mo (K5007, Dako Denmark A/S, Denmark) were then utilized to detect the bound antibody. A total of six fields were randomly selected and measured semi-quantitatively using ImageJ software (National Institutes of Health, Bethesda, MD).

\section{Quantitative real-time PCR}

Placental tissue was ground in liquid nitrogen, and then total RNA was extracted with TRIzol reagent (Invitrogen, Waltham, MA) following the manufacturer's instructions. Microspectrophotometry and gel electrophoresis were used to test the purity and integrity of RNA. For PCR of mRNA, RNA was reverse transcribed using HiScript III RT SuperMix (Vazyme, Nanjing, China). qPCR was performed using ChamQ SYBR qPCR Master Mix (Vazyme, Nanjing, China) on the LC480 system (Roche, La Jolla, CA, USA). The specific primer was listed in the data supplementary. The relative expression slevels were calculated using the $2^{(-\Delta \Delta C T)}$ method.

\section{Cell culture}

TEV-1, a human extravillous trophoblast cell line, was a gift from The University of Hong Kong and The Chinese University of Hong Kong. Cells were cultured in Dulbecco's modified Eagle's medium: DMEM/F12 supplemented with $10 \%$ foetal bovine serum and $1 \%$ penicillin/ streptomycin (Gibco; Thermo Fisher Scientific, Waltham, MA, USA) in an incubator containing 5\% $\mathrm{CO}_{2}$ at $37^{\circ} \mathrm{C}$.

\section{Western blot}

EVT-1 cells were treated with LPS for $24 \mathrm{~h}$, whole lysates were prepared by direct lysis in RIPA buffer with PMSF (Beyotime, Beijing, China). Electrophoresis processes were performed as previously described [18]. Primary antibodies of CLU (DF6421, Affinity, Cincinnati, OH, USA) and GAPDH (AF7021, Affinity, Cincinnati, $\mathrm{OH}, \mathrm{USA}$ ) were used in this analysis. Antigens were revealed using a chemiluminescence assay (Affinity, Cincinnati, OH, USA). Quantification of bands was achieved by densitometry using the Chemiluminescence Image analysis system (GE Healthcare, BI600, Boston, MA, USA).

\section{Matrigel transwell assay}

TEV-1 cells were starved for $12 \mathrm{~h}$, digested and resuspended in serum-free DMEM/F12 $\left(1 \times 10^{5}\right.$ cell $\left./ \mathrm{ml}\right)$. Transwell chambers for the invasion assay were precoated with Matrigel. Two hundred microlitres of cell mix was added to the upper chamber, and then $500 \mu \mathrm{L}$
DMEM/F12 medium supplemented with 20\% FBS was added to the lower chamber. In addition, the lower chamber contained recombinant human CLU (R\&D Systems, Minneapolis, Minnesota, USA) at final concentrations of $0,5,10,20,50$, and $100 \mathrm{ng} / \mathrm{ml}$. After $24 \mathrm{~h}$, cells were fixed with $4 \%$ paraformaldehyde for $20 \mathrm{~min}$ and stained with $0.1 \%$ crystal violet for $10 \mathrm{~min}$, and then images were captured with a microscope. The level of cell invasion was quantified by averaging the total number of cells in three random fields. The experiment was repeated three times.

\section{Statistical analysis}

The chi-square test was used to compare categorical variables between patients and controls. Independent $t$-tests were used to compare the differences between continuous variables. Differentially expressed proteins were determined using fold change $>1.2$ and $P$-value $<0.05$. A volcano plot was generated using $\mathrm{R}$ software (version 3.6.2) with the ggplot2 package, and the PPI network was constructed using the STRING database (https:// string-db.org) and Cytoscape software (version 3.7.2). Logistic regression was used to estimate the odds ratio (OR), and the ROC curve was used to evaluate the model prediction capability. To compare quantitative variables of serum proteins and mRNA expression of placenta, Student's $t$-test and one-way ANOVA were used where appropriate. Data were analysed with SPSS (version 22.0; IBM Inc., city NY, USA). A two-tailed $P<$ 0.05 was considered statistically significant.

\section{Results}

\section{Clinical characteristics}

The features of the study participants are presented in Table 1. The demographics, medical history, and newborns of healthy and pre-HDP pregnancies were compared. Women with an elevated body mass index (BMI) before 20 weeks of gestation were more likely to develop HDP, and there was foetal growth restriction in the HDP group $(P<0.05)$.

\section{Screening set}

Based on label-free LC-MS/MS, we identified a total of 769 serum proteins in the screening set $(n=24)$; among them, 33 proteins were upregulated, and 14 were downregulated (Fig. 1a). Pearson correlation analysis was used in the heatmap to cluster the proteins and samples separately, and this showed that these 47 proteins could be divided into four categories, which could distinguish the disease group from the control group (Fig. 1b). The biological process, cellular component, and KEGG (top 6) analyses showed that inflammation activation and the complement system might play a key role in HDP 
Table 1 Characteristics of pre-HDP patients and healthy pregnancies

\begin{tabular}{|c|c|c|c|}
\hline \multirow[t]{3}{*}{ Factors } & \multicolumn{3}{|c|}{ The screening and validation set } \\
\hline & Control $(n=148)$ & Pre-HDP $(n=148)$ & $P$ \\
\hline & $\mathrm{N}(\%)$ & N (\%) & \\
\hline \multicolumn{4}{|l|}{ Demographic characteristics } \\
\hline Han nationality, n (\%) & $145(97.97)$ & $145(97.97)$ & 1.00 \\
\hline Maternal age (years) & $30.84 \pm 4.46$ & $30.99 \pm 4.69$ & 0.83 \\
\hline Weight (kg) & $64.71 \pm 9.16$ & $72.12 \pm 11.44$ & $<0.001$ \\
\hline BMI $\left(\mathrm{kg} / \mathrm{m}^{2}\right)$ & $25.37 \pm 2.47$ & $28.49 \pm 3.98$ & $<0.001$ \\
\hline Diastolic ( $\leq 20$ wk) & $73.54 \pm 12.94$ & $87.9 \pm 12.96$ & $<0.001$ \\
\hline Systolic ( $\leq 20$ wk) & $117.4 \pm 17.63$ & $138.39 \pm 15.60$ & $<0.001$ \\
\hline GA at sample collection & $14.27 \pm 4.80$ & $14.36 \pm 4.64$ & 0.917 \\
\hline GA at delivery & $39.02 \pm 0.95$ & $37.45 \pm 2.39$ & $<0.001$ \\
\hline \multicolumn{4}{|l|}{ Medical history } \\
\hline Anaemia n (\%) & $14(10.22)$ & $24(18.46)$ & 0.054 \\
\hline Family history of DM, n (\%) & $2(1.47)$ & $6(4.35)$ & 0.16 \\
\hline Family history of HDP, n (\%) & $1(0.74)$ & $2(1.45)$ & 0.57 \\
\hline \multicolumn{4}{|l|}{ Past obstetric history } \\
\hline Gravidity $\geq 2, n(\%)$ & $93(62.84)$ & 95 (64.19) & 0.81 \\
\hline History of term delivery $\geq 1, \mathrm{n}(\%)$ & $117(79.05)$ & $106(71.62)$ & 0.14 \\
\hline Spontaneous miscarriage $\geq 1, \mathrm{n}(\%)$ & $21(14.19)$ & $26(17.57)$ & 0.43 \\
\hline
\end{tabular}

GA gestational age, $B M I$ body mass index, DBP diastolic blood pressure, SBP systolic blood pressure, DM diabetes disease, HDP hypertension disorder of pregnancy. Data are presented as the mean \pm SD (standard deviation) and analysed by Student's $t$-test. Data are presented as $n$ (\%) and were analysed using a chi-square test or Fisher's exact test. Women who developed HDP and women who had a healthy pregnancy were matched by age and gestational week

development (Fig. 1c). To further capture the relationships between the terms, a subset of enriched terms was selected and rendered as a network plot, where terms with a similarity $>0.3$ were connected by edges. The terms with the best $p$-value were selected from 20 clusters, each cluster was limited to no more than 15 terms, and the total did not exceed 250 terms. Pathway cluster analysis further indicated the importance of complement system activation, which involved platelet degranulation, wound healing, and inflammatory responses (Fig. 1d).

\section{First verification phase}

In the first verification phase, the sample size was expanded, and the same methodology was repeated with a focus on the complement proteins. The verification data were consistent with those of the screening phase by 93.5\% (Table. S2). In the first verification phase, a total of eight proteins associated with the complement system (FC $>1.2 ; P<0.05$ ), including CLU, fibrinogen gamma chain $(\mathrm{FIB}-\gamma)$, complement factor $\mathrm{H}$-related protein 5 (CFHR5), complement $\mathrm{C} 8$ beta chain (C8 $\beta)$, complement $\mathrm{C} 1 \mathrm{~s}(\mathrm{C} 1 \mathrm{~s})$, complement $\mathrm{C} 1$ inhibitor (C1-INH), fibronectin (FN), and C-reactive protein (CRP), were significantly different (Fig. 2a).
The CLU level was significantly increased in preHDP pregnancies $(F C=1.96, P=0.0004)$ and was positively correlated with disease severity (FC was 0.75 for $\mathrm{GH}$ vs. 4.39 for PE). Pearson's correlation demonstrated that CLU had a strong positive relationship with FIB- $\gamma(r=0.937)$ and CFHR5 $(r=0.847)$, which indicated that their role in pre-HDP might be highly relevant (Fig. 2a). Besides, we evaluated the relationship between these clinical features and candidate markers because there are large differences in patient's baseline weight, blood pressure, and anaemia (Table 1 and Table 2). Bodyweight, systolic blood pressure (SBP), diastolic blood pressure (DBP), and haemoglobin concentration (HGB, represent the degree of anaemia) have no-significance relationships with most of these eight proteins, and C1-INH was negatively correlated with DBP (Fig. 2b). Via PPI analysis, CLU was a critical factor for the development of HDP and had a function in the membrane attack complex (C5b-9, MAC), while FIB- $\gamma$ and CFHR5 showed a correlation with $\mathrm{C} 3$ (Fig. 2c). C8 $\beta, \mathrm{C} 1$ s, and $\mathrm{C} 1-\mathrm{INH}$ showed a low correlation with other proteins. We further analysed whether these proteins were expressed differently in pre-GH and pre-PE pregnancies. The CLU, FIB- $\gamma$, CFHR5, FN, and CRP levels in pre-PE pregnancies were higher than those in the pre-GH group (Fig. 2d). 


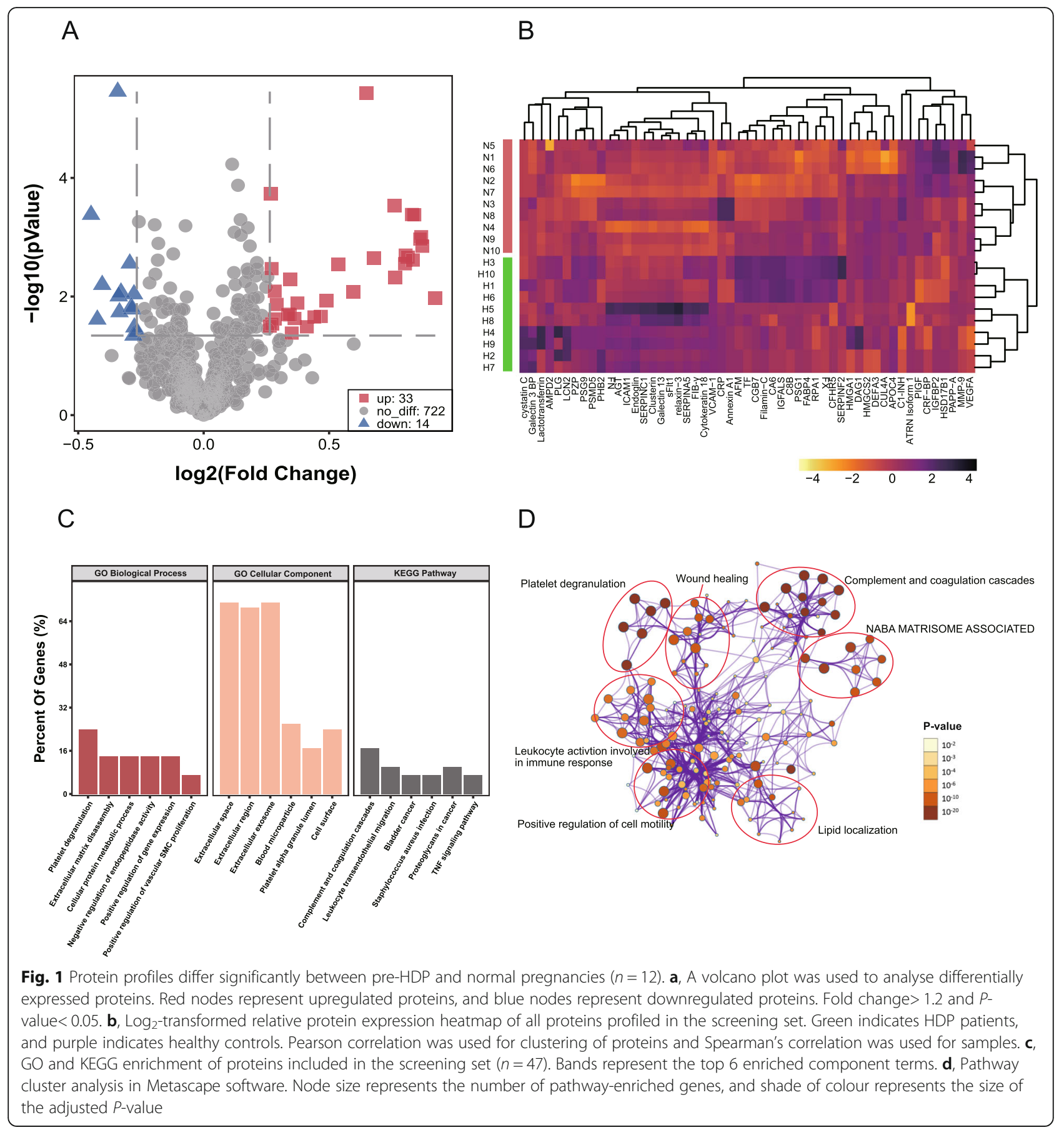

\section{Model construction}

In the first verification phase, the capability of models of these eight proteins to predict HDP was determined by ROC analysis (Fig. 3). The results showed that some of the proteins have a potential value to discriminate before clinical symptoms occur. For HDP prediction, the AUCs were C8 $\beta$ (0.77), CLU (0.77), CFHR5 (0.71), CRP (0.74), FIB- $\gamma$ (0.73), C1s (0.74), FN (0.73), C1-INH (0.77) with $P<0.05$ for all these values. Some clinical characteristics also showed potential predictive ability, such as body weight (0.68), SBP (0.69), DBP (0.75) and HGB (0.58), and their ROC curves were concluded in Fig. S3.

A predictive model was then developed through logistic regression based on the clinical characteristics and the candidate markers. When CLU, CFHR5, C8 $\beta$, CRP, and body weight were used in combination, the capability of HDP prediction was the highest, which AUC increase to 0.92 (95\% CI $0.83-0.99)$ with the sensitivity of 0.80 , and the specificity of 0.88 (Fig. 3a). Moreover, in the analysis of HDP subtype, the AUC of combined 


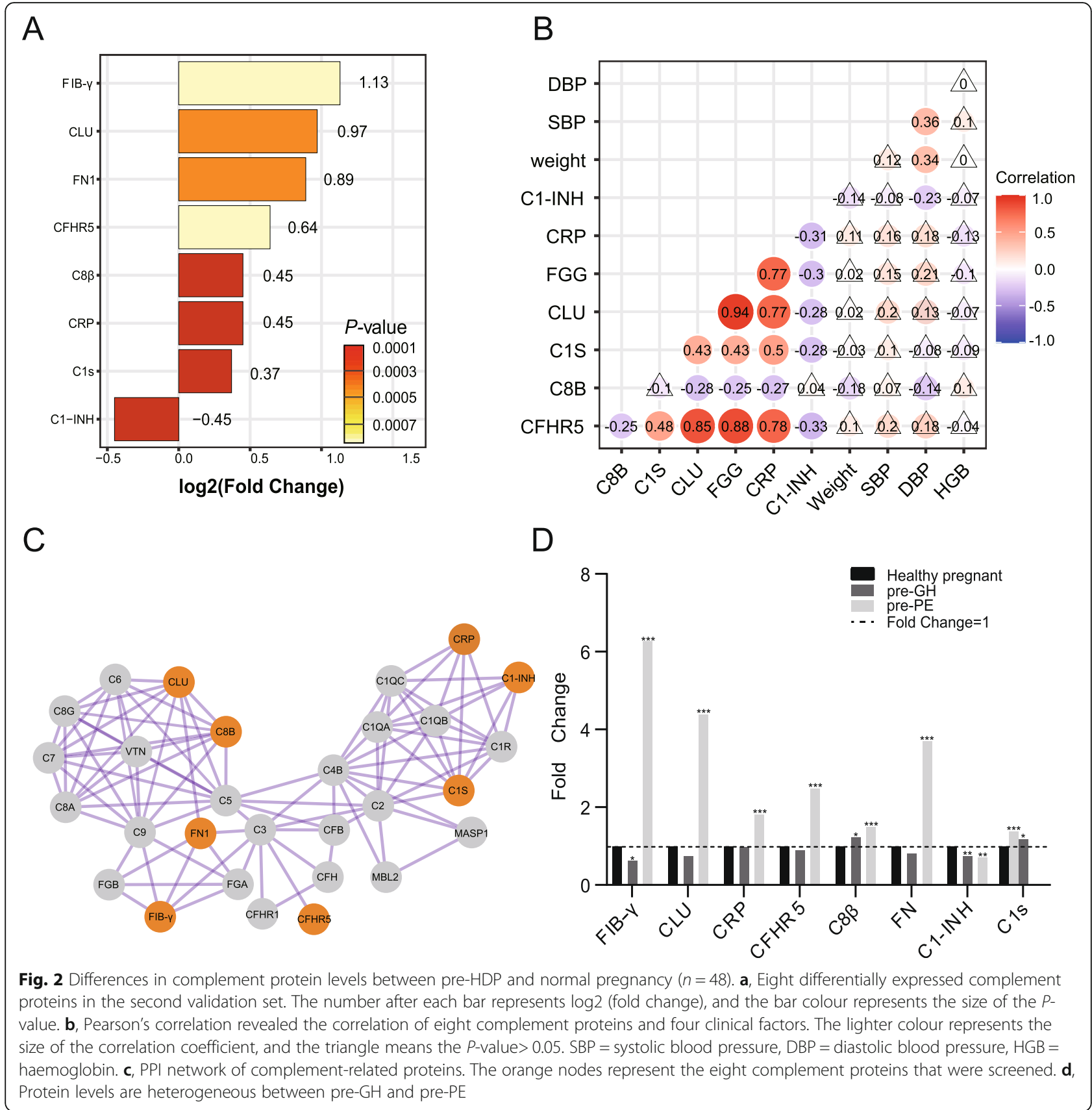

CLU, CFHR5, C8 $\beta$, and CRP for GH prediction was 0.84 (95\% CI $0.63-0.92)$ with a sensitivity of 0.78 and specificity of 0.89 (Fig. 3b), and for PE, it was 0.96 (95\% CI 0.94-1.00) with a sensitivity of 0.87 and specificity of 0.92 (Fig. 3c).

\section{Model validation}

In the second verification phase, the efficacy of this model was validated in another independent cohort (including 29 pre-GH, 59 pre-PE, and 88 normal pregnancies). ELISA kits were used due to their high repeatability and specificity. Serum CLU, CFHR5, and
CRP increased in the pre-HDP groups compared with the normal pregnancies (Fig. 4a-c). Compared to those in normal pregnancies, CLU $(469.71 \pm 211.53$ vs. $349.49 \pm 169.59 \mu \mathrm{g} / \mathrm{ml}, \quad$ OR $=1.22, \quad P<0.001), \quad$ CFHR5 $(17.49 \pm 4.65$ vs. $14.78 \pm 3.76 \mathrm{ng} / \mathrm{ml}, \quad$ OR $=1.13, \quad P<$ $0.001)$, and CRP $(4.99 \pm 6.03$ vs. $3.48 \pm 3.51 \mathrm{mg} / \mathrm{L}, \mathrm{OR}=$ $1.27, P=0.04)$ were increased in pre-HDP pregnancies. The levels of CLU $(416.54 \pm 236.07$ vs. $337.59 \pm$ $166.76 \mu \mathrm{g} / \mathrm{ml}, P<0.001)$ and CFHR5 (17.26 \pm 5.00 vs. $14.11 \pm 3.70 \mathrm{ng} / \mathrm{ml}, \quad P<0.001)$ in pre-GH pregnancies were higher than those in normal pregnancies, but there was no difference in CRP $(3.40 \pm 6.07$ vs. $2.90 \pm 3.25 \mathrm{mg} /$ 
Table 2 Maternal adverse outcomes of pre-HDP patients and healthy pregnancies

\begin{tabular}{|c|c|c|c|}
\hline \multirow[t]{3}{*}{ Factors } & \multicolumn{3}{|c|}{ The screening and validation set } \\
\hline & Control $(n=148)$ & Pre-HDP $(n=148)$ & $P$ \\
\hline & $\mathrm{N}(\%)$ & N (\%) & \\
\hline \multicolumn{4}{|l|}{ Maternal adverse outcomes } \\
\hline Parturient at admission, $\mathrm{n}(\%)$ & $126(86.7)$ & $102(73.2)$ & 0.001 \\
\hline PROM, n (\%) & $32(24.06)$ & $27(19.57)$ & 0.97 \\
\hline Umbilical cord around neck, n (\%) & $26(19.12)$ & $21(15.79)$ & 0.47 \\
\hline Caesarean section, n (\%) & $6(4.42)$ & $61(45.86)$ & $<0.001$ \\
\hline Postpartum haemorrhage, n (\%) & $2(1.47)$ & $0(0)$ & 0.16 \\
\hline Oedema of lower limbs, n (\%) & $3(2.17)$ & $18(13.53)$ & 0.08 \\
\hline Soreness of waist, n (\%) & $0(0)$ & $7(5.26)$ & / \\
\hline Cramps, n (\%) & $0(0)$ & $0(0)$ & / \\
\hline \multicolumn{4}{|l|}{ Infant } \\
\hline Birth weight (g) & $3196.15 \pm 320.21$ & $2823.69 \pm 680.40$ & $<0.001$ \\
\hline \multicolumn{4}{|l|}{ Paternal condition } \\
\hline Paternal age (years) & $31.72 \pm 6.06$ & $31.52 \pm 5.83$ & 0.81 \\
\hline
\end{tabular}

$\mathrm{L}, P=0.012)$. In addition, the levels of CLU (492.76 \pm 232.95 vs. $354.49 \pm 170.44 \mu \mathrm{g} / \mathrm{ml}, \quad P<0.001), \quad$ CFHR5 $(17.52 \pm 5.08$ vs. $15.07 \pm 3.77 \mathrm{ng} / \mathrm{ml}, P<0.001)$, and CRP $(5.82 \pm 6.03$ vs. $3.68 \pm 3.51 \mathrm{mg} / \mathrm{L}, P=0.039)$ were higher in pre-PE pregnancies than in normal pregnancies.

The AUC for the HDP prediction model of CLU combined with CFHR5, CRP and body weight was 0.74 with a sensitivity of 0.67 and specificity of 0.68 (Fig. 4d). For GH prediction, the AUC was 0.72 with a sensitivity of 0.60 and specificity of 0.72 (Fig. 4e). For PE, the AUC was 0.77 with a sensitivity of 0.79 and specificity of 0.73 for PE prediction (Fig. 4f).

\section{Localization and expression of CLU in the placenta} CLU is immunolocalized extravillous trophoblasts (EVTs), syncytiotrophoblasts (STBs), and cytotrophoblasts (CTBs) of the placenta (Fig. 5a). The CLU immunostaining intensity was higher than that in normal pregnancies, while CLU mRNA expression was higher in $\mathrm{PE}$ placentas than in healthy controls (Fig. 5b).

\section{LPS promotes CLU expression}

The CLU expression of TEV-1 cells was significantly upregulated following treatment with $100 \mathrm{ng} / \mathrm{ml}$ and 500

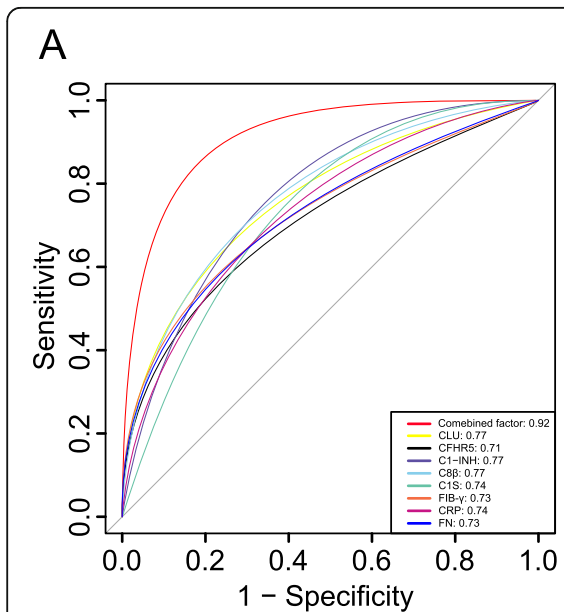

B

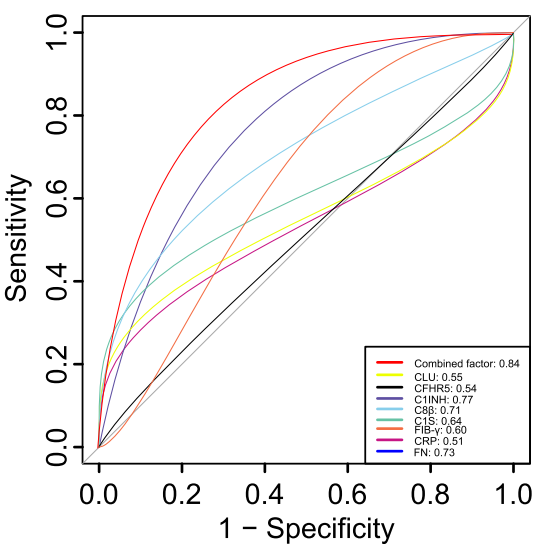

C

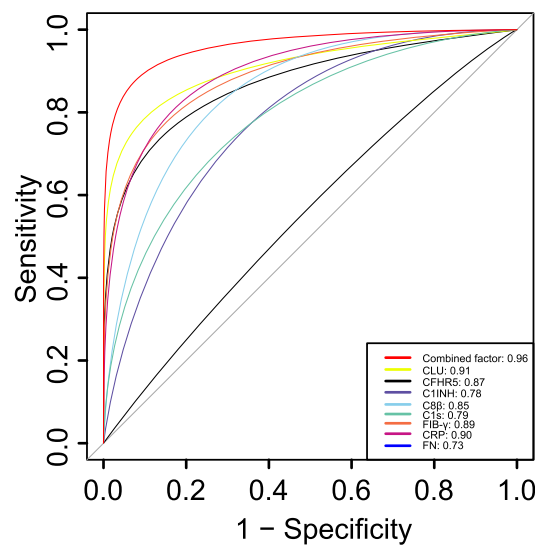

Fig. 3 A, Receiver operating curve (ROC) of individual and combined markers for HDP by LC-MS/MS $(n=48)$. B, ROC curve of markers for GH prediction $(n=22)$. C, ROC curve of markers for PE prediction $(n=26)$. The area under the curve (AUC) for each curve is listed in the lower right corner. HDP: hypertension disorder of pregnancy, GH: gestational hypertension, PE: preeclampsia 


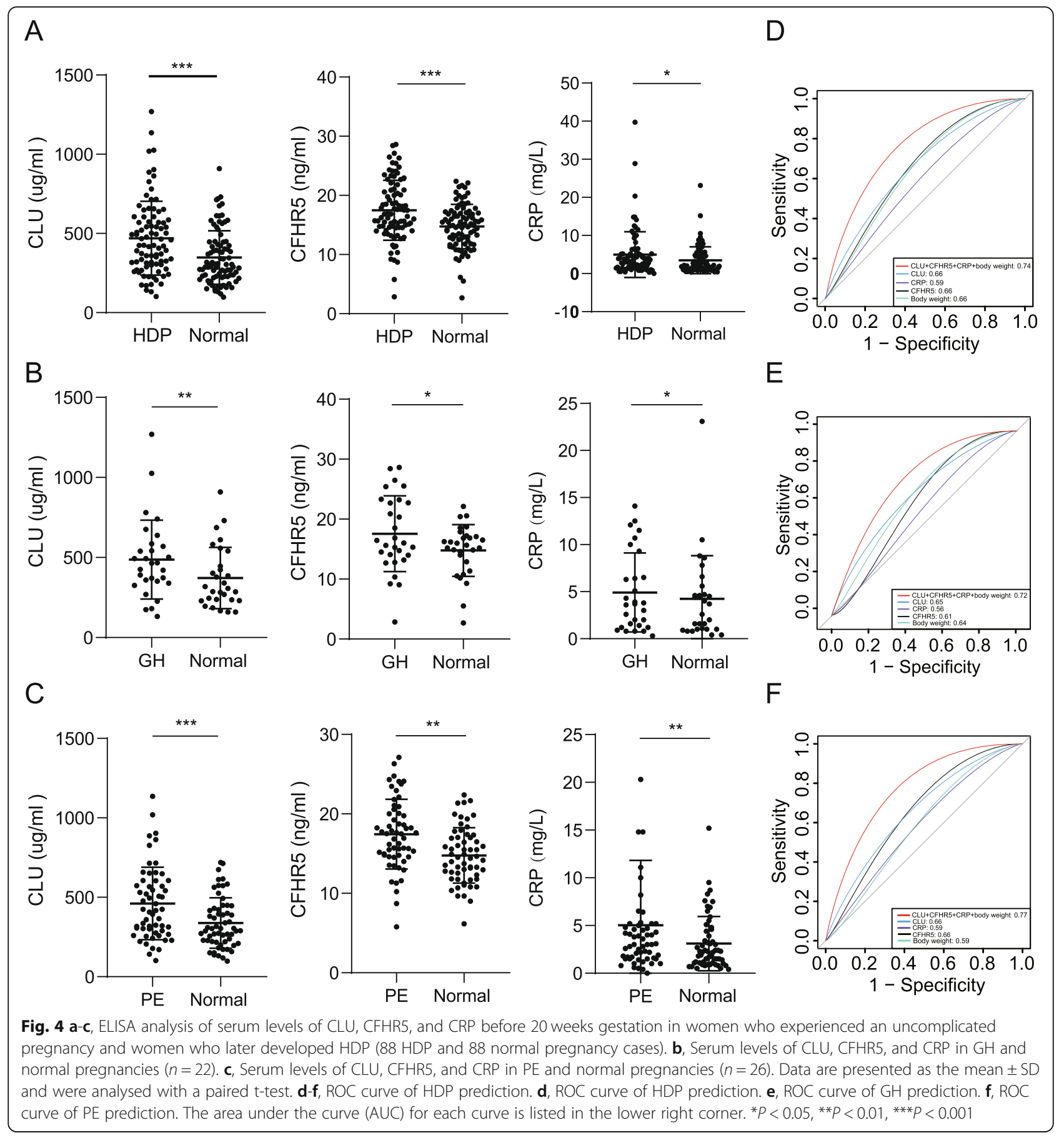

ng/ml LPS $(P=0.001)$ (Fig. 5b). And CLU mRNA level is positively correlated with LPS concentration (Fig. S2).

\section{CLU inhibits invasion of EVT}

The expression of MMP9 mRNA was downregulated in the placentas of pregnant women with $\mathrm{PE}$ and negatively correlated with CLU mRNA (Fig. 5c, d). TEV-1 cell invasion was inhibited by treatment with
$50 \mathrm{ng} / \mathrm{ml}$ and $100 \mathrm{ng} / \mathrm{ml} \mathrm{CLU}$, as determined by the invasion assay $(P<0.001$, Fig. $5 \mathrm{e})$.

\section{Discussion}

In the current study, the difference in serum proteins between pre-HDP and normal pregnancies was identified by a label-free nano LC-MS/MS method, and 47 differential proteins were found. Subsequently, a novel prediction model for HDP was constructed, and it 


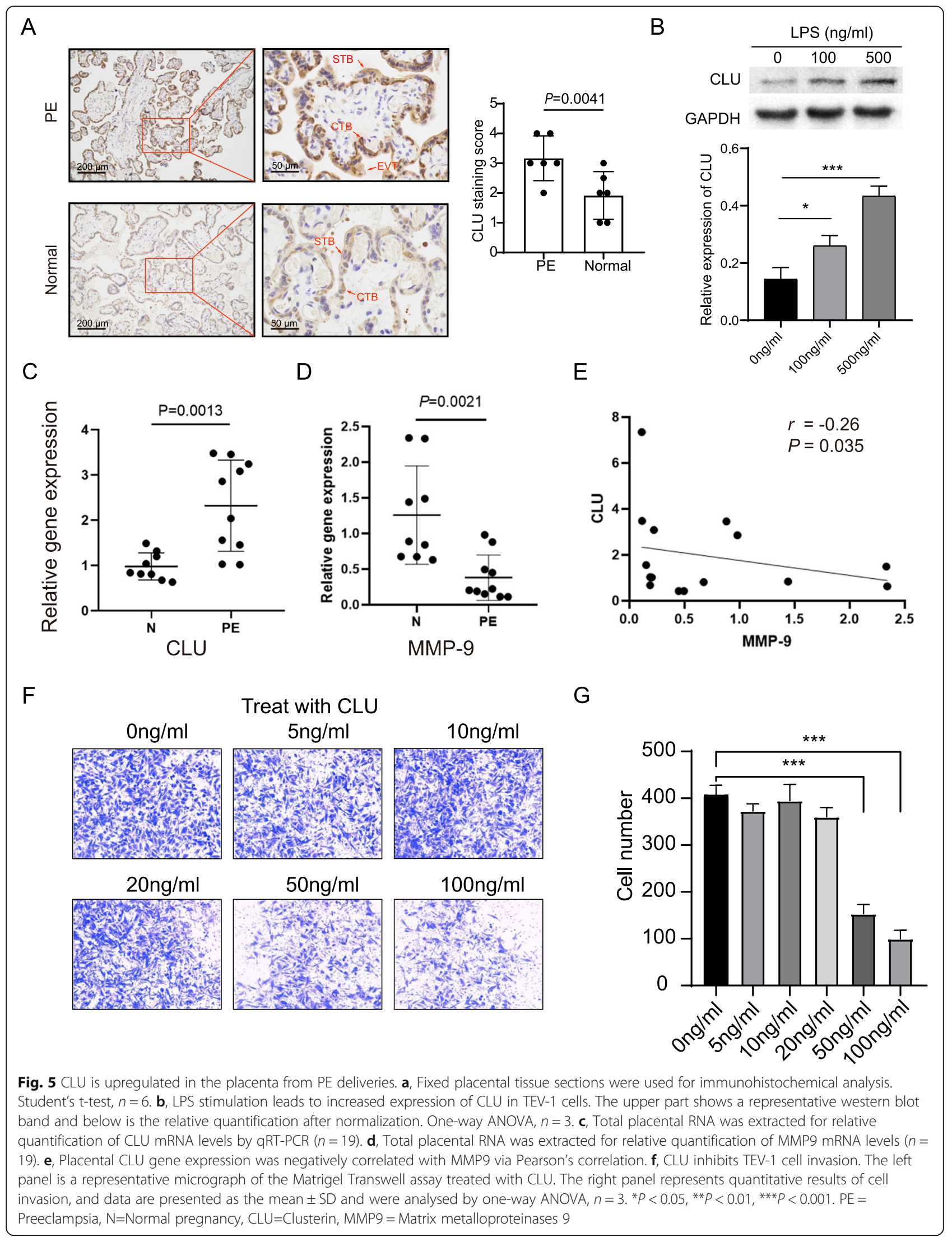


was validated in another independent cohort. Additionally, in in vitro assays, CLU inhibited the invasion of TEV-1 cells.

Increasing studies have highlighted that complement system disorders are related to the occurrence and development of HDP and that complement molecules may be potential markers for PE screening and diagnosis [11]. The results of the current study also showed that in pre-HDP, there is disorder among components of the complement system that are involved in the complement classical pathway (CRP and C1s), complement membrane attack $(C 8 \beta)$, and complement regulation (CLU, CFHR5, and C1-INH). To the best of our knowledge, this is the first study to find that pre-HDP pregnancies have elevated levels of CFHR5 and C8 $\beta$. Previous studies have found that complement factors $\mathrm{Bb}$ and $\mathrm{C} 5 \mathrm{a}$ are negatively correlated with placental growth factor (PIGF), leading to incomplete spiral artery remodeling and endothelial dysfunction $[19,20]$. Kim and Kolla also found that C1s and FIB- $\gamma$ levels were increased in PE [21, 22]. Researchers believe that the increase in placenta-derived debris and apoptotic granules in the circulation of HDP pregnancies is the leading cause of the activation of the complement classical pathway and alternative pathway [23]. Excessive dysregulation of the complement system may lead to the insufficient clearance of apoptotic granules and placenta-derived debris and result in excessive inflammation, which may lead to the typical symptoms of HDP (proteinuria and new-onset hypertension) [11]. Mutations in the complement inhibitor gene can also cause HELLP syndrome (a severe subtype of HDP) [23-25]. Moreover, Burwick and colleagues successfully treated patients with HELLP syndrome with eculizumab (a C5 targeted inhibitor), which showed that suppressing complement activation could improve HDP symptoms [26].

Disorder of complement regulatory factors (CLU, CFHR5, and C1-INH) may be associated with excessive inflammation and visceral organ injury in mothers with HDP. CFHR5, an analogue of complement factor $\mathrm{H}$, can enhance local AP activation via interference with the C-inhibiting function of factor $\mathrm{H}$ in the cell membrane $[27,28]$. Existing studies have not found an association with pregnancy-related diseases, but CFHR5 is positively associated with kidney injury. CFHR 5 mutation was considered the cause of atypical haemolytic uraemic syndrome [29]. Through the mechanism of kidney injury, we speculate that elevated CFHR5 causes excessive AP activation, which leads to vascular endothelial damage and the release of inflammatory factors, ultimately resulting in PE. On the other hand, this suggests that renal impairment may occur before blood pressure increases.
Our team considered that CLU might be a critical biomarker for the prediction of the development of HDP. CLU is a secreted glycoprotein, founding in various body fluids, which has multiple functions, including regulating apoptosis, translocating lipids, inhibiting complement, regulating immunity, and cell invasion [30]. Some literature highlighted that CLU was associated with pregnancy diseases, such as intrauterine growth restriction, recurrent pregnancy loss, and small gestational age [31-33]. There are some intersecting pathophysiological mechanisms between HDP and intrauterine growth restriction and recurrent miscarriage involved in placental implantation and development dysfunction [34]. However, the role of CLU in placental development and trophoblast differentiation is still unclear. It is currently recognized that insufficient trophoblast invasion and lack of differentiation are part of the pathogenesis of HDP [35]. Previous research has reported that CLU could regulate the proliferation and invasion of vascular smooth muscle and tumor [15, 36, 37]. The current study shows that CLU characteristics are interesting in the pathogenesis of HDP. First, the fact that serum CLU was positively correlated with the severity of HDP, and it was mainly localized to CTBs at the HDP placenta, suggests that it is important in the development of HDP and the differentiation of trophoblast cells. Second, we demonstrated that the invasion ability of EVTs was reduced by treating CLU, which expression increased was related to LPS treatment. Therefore, inflammationinduced CLU could inhibit EVT's invasion, which further may cause insufficient spiral artery remodeling and placental ischemia. Eventually, it will lead to increased maternal oxidative stress and cause systemic symptoms. Besides, compared with other candidates in this work, although the CLU increase is not the highest, it plays an important role in regulating oxidative stress and cell apoptosis [30]. The occurrence and development of HDP involved excessive oxidative stress and apoptosis too [35]. So, we think it is interesting to study CLU's effect on oxidative stress and apoptosis in HDP, and these hypotheses are worthy of further exploration.

We found that LPS-induced inflammation increases CLU expression, indicating CLU elevation is a downstream response induced by excessive inflammation. In HDP, the inflammatory response is inflated due to aberrant activation of innate immune cells and imbalanced differentiation of $\mathrm{T}$-helper cell subsets $[11,38]$. In this study, serum FN, FIB- $\gamma$ and CRP were more abundant in HDP pregnancies than normal pregnancies. Previous studies have found that pre-PE pregnancies have more abundant FN, FIB- $\gamma$ and CRP levels involved in excessive inflammation and organ damage [39-42]. CLU level may involve inflammation-induced organ damage because it's highly correlated with inflammation markers (FN, FIB- $\gamma$, and CRP). 
Evaluation of the performance of markers indicated that the combination of multiple factors can provide a better prediction model than any of the individual proteins alone for predicting HDP. In the screening cohort, ROC analysis showed that individual panels comprising C8 $\beta$, CLU, CFHR5, CRP, FIB- $\gamma, \mathrm{C} 1 \mathrm{~s}$, FN, and C1-INH performed well in predicting $\mathrm{GH}$ and $\mathrm{PE}$. Logistic regression was used to select possible risk factors from clinical characteristics and candidate markers to develop a predictive model. The highest predictive capability model was then generated for HDP (CLU, CFHR5, C8 $\beta$, CRP, and body weight). This model was subsequently verified in another cohort. Compared with those in normal pregnancies, these three proteins' levels increased in pre-HDP pregnancies with the ELISA method. The combination of CLU, CRP, CFHR5, and body weight for prediction of HDP has an AUC of 0.74, a sensitivity of 0.67, and a specificity of 0.68 . Compared with LC-MS/MS approach, although the AUC decreases in the ELISA method, the validation model still has considerable prediction efficiency. Especially for PE, the model has a higher prediction efficiency, which is important for distinguish PE from GH. And the model is independent of the clinical characteristics, including blood pressure and body weight, which was not related to elevated candidate biomarkers. Due to the exhaustion of specimens, only CLU, CRP, and CFHR5 were verified; otherwise, this model would be more reliable if $C 8 \beta$ was also added.

This study has some limitations. Compared with the clinical environment, the small sample size may cause differences in sensitivity and specificity. Thus, we will expand the research object in the next study and conduct a multicentre study to further improve the model. The role of CLU in HDP development needs to be further verified in appropriate in vitro and in vivo models.

\section{Conclusions}

In summary, we consider that there are dysregulated classical pathways and alternative pathways of the complement system, leading to excessive inflammation and endothelial damage, which is associated with the development of HDP. Moreover, CLU can inhibit EVT cell invasion in vitro. The combination of CLU, CFHR5, C $8 \beta$, CRP, and body weight might improve the early prediction of HDP.

\footnotetext{
Abbreviations

HDP: Hypertensive disorder of pregnancy; GH: Gestational hypertension; PE: Preeclampsia; CLU: Clusterin; MMP9: Matrix metalloproteinases 9; GAPD $\mathrm{H}$ : Glyceraldehyde-3-phosphate dehydrogenase; OR: Odds ratio; BMl: Body Mass Index; FIB- $\gamma$ : Fibrinogen gamma chain; CFHR5: Complement factor $\mathrm{H}$ related protein 5; C8ß: Complement $\mathrm{C} 8$ beta chain; $\mathrm{C} 1 \mathrm{~s}$ : Complement C1s; C1-INH: Complement C1 inhibitor; FN: Fibronectin; CRP: C-reactive protein; PPI: Protein-protein interaction; MAC: Membrane attack complex; STB: Syncytiotrophoblast; PIGF: Placental growth factor; EVT: Extravillous trophoblast; ROC: Receiver operating characteristic curve; AUC: Area Under Curve
}

\section{Supplementary Information}

The online version contains supplementary material available at https://doi. org/10.1186/s12958-021-00742-z.

\section{Additional file 1.}

\section{Acknowledgements}

Not applicable.

\section{Authors' contributions}

Shanshui Zeng, Wenjin Fu, and Hongling Yang conceived and directed the study; Shanshui Zeng, Mengru Han, and Ming Jiang did the analysis and visualization; Shanshui Zeng, Mengru Han, Fei Liu, Min Jiang, Yanwei Hu, Yan Long, Fangling Zeng, Qiangsheng Gan, and Weitao Ye collected the samples: Shanshui Zeng and Mengru Han drafted the manuscript; Yanwei Hu, and Hongling Yang, Chunyan Zhu revised the manuscript. The author(s) read and approved the final manuscript.

\section{Funding}

This study was funded by the National Natural Science Fund of China (81871716), the Natural Science Fund of Guangdong Province (2018A0303130314), the Science and Technology project Fund of Guangzhou (201704020109, 201707010019), the fund from Guangzhou Institute of Pediatrics/Guangzhou Women and Children' s Medical Center (NO: IP-2019-009), and fund from Guangzhou Women and Children's Medical Center (No. CWCMC2020-6-017)

Availability of data and materials

All data are included in the article.

\section{Declarations}

Ethics approval and consent to participate

This study is reviewed and approved by the Ethics Committee of Guangzhou Women and Children's Medical Center (Ethics number: 2018030306) with informed consent of the patients.

\section{Consent for publication}

All the authors review the manuscript and consent for publication.

\section{Competing interests}

The authors declare that they have no competing interests.

\section{Author details}

'Department of Laboratory, Guangzhou Women and Children's Medical Centre, Guangzhou Medical University, No.9, Jinsui Road, Guangzhou 510623, Guangdong, China. ${ }^{2}$ School of Public Health, Guangzhou Medical University, Guangzhou 511436, China. ${ }^{3}$ Department of Gynaecology and Obstetrics, Guangzhou Women and Children's Medical Centre, Guangzhou Medical University, Guangzhou 510623, China. ${ }^{4}$ Clinical Laboratory, Houjie Hospital of Guangdong Medical University, HeTian Road, Dongguan 523945,

Guangdong, China.

Received: 18 February 2021 Accepted: 7 April 2021

Published online: 19 April 2021

References

1. Umesawa M, Kobashi G. Epidemiology of hypertensive disorders in pregnancy: prevalence, risk factors, predictors and prognosis. Hypertens Res. 2017;40(3):213-20. https://doi.org/10.1038/hr.2016.126.

2. Behrens I, Basit S, Melbye M, Lykke JA, Wohlfahrt J, Bundgaard H, et al. Risk of post-pregnancy hypertension in women with a history of hypertensive disorders of pregnancy: nationwide cohort study. BMJ. 2017;358:3078.

3. Mol BWJ, Roberts CT, Thangaratinam S, Magee LA, de Groot CJM, Hofmeyr GJ. Pre-eclampsia. Lancet. 2016;387(10022):999-1011. https://doi.org/10.101 6/50140-6736(15)00070-7.

4. ACOG Practice Bulletin No. 202: gestational hypertension and preeclampsia. Obstet Gynecol. 2019;133:e1-25. 
5. US Preventive Services Task Force, Bibbins-Domingo K, Grossman DC, Curry SJ, Barry MJ, Davidson KW, et al. Screening for Preeclampsia: US Preventive Services Task Force Recommendation Statement. JAMA. 2017;317:1661-7.

6. Nobakht M, Gh BF. Application of metabolomics to preeclampsia diagnosis. Syst Biol Reprod Med. 2018;64:324-39. https://doi.org/10.1080/19396368.201 8.1482968 .

7. Borzychowski AM, Sargent IL, Redman CWG. Inflammation and preeclampsia. Semin Fetal Neonatal Med. 2006;11:309-16. https://doi.org/10.101 6/j.siny.2006.04.001.

8. Yang $H$, Tang W, Zhu C, Guo C. Platelets, inflammation, and prediction of the hypertension disorders of pregnancy. J Matern Fetal Neonatal Med. 2012;25:99-103. https://doi.org/10.3109/14767058.2011.560985.

9. Yang H, Wang Q, Tang W, Zhu C, Xiao Y, Wang J. The predictive value of leukocyte parameters for hypertensive disorders of pregnancy in South China. Hypertension in Pregnancy. 2012;31:11-21. https://doi.org/10.3109/ 0641955.2010 .507836$.

10. Ma Y, Kong L-R, Ge Q, Lu Y-Y, Hong M-N, Zhang Y, et al. Complement 5amediated trophoblasts dysfunction is involved in the development of preeclampsia. J Cell Mol Med. 2018;22:1034-46. https://doi.org/10.1111/ jcmm.13466.

11. Teirilä L, Heikkinen-Eloranta J, Kotimaa J, Meri S, Lokki Al. Regulation of the complement system and immunological tolerance in pregnancy. Semin Immunol. 2019;45:101337. https://doi.org/10.1016/.smim.2019.101337.

12. Geyer PE, Holdt LM, Teupser D, Mann M. Revisiting biomarker discovery by plasma proteomics. Mol Syst Biol. 2017;13:942. https://doi.org/10.15252/ msb.20156297.

13. Murphy MS-Q, Bytautiene E, Saade G, Smith GN. Alterations to the maternal circulating proteome after preeclampsia. Am J Obstetr Gynecol. 2015;213: 853.e1-9.

14. Blankley RT, Fisher C, Westwood M, North R, Baker PN, Walker MJ, et al. A label-free selected reaction monitoring workflow identifies a subset of pregnancy specific glycoproteins as potential predictive markers of earlyonset pre-eclampsia. Mol Cell Proteomics. 2013;12:3148-59. https://doi.org/1 0.1074/mcp.M112.026872.

15. Muhammad LA, Saad F. The role of clusterin in prostate cancer: treatment resistance and potential as a therapeutic target. Expert Rev Anticancer Ther. 2015;15:1049-61. https://doi.org/10.1586/14737140.2015.1064769.

16. Pucci S, Greggi C, Polidoro C, Piro MC, Celi M, Feola M, et al. Clusterin silencing restores myoblasts viability and down modulates the inflammatory process in osteoporotic disease. J Transl Med. 2019:17:118. https://doi.org/1 0.1186/s12967-019-1868-5.

17. Zhou Y, Zhou B, Pache L, Chang M, Khodabakhshi AH, Tanaseichuk O, et al. Metascape provides a biologist-oriented resource for the analysis of systems-level datasets. Nat Commun. 2019;10:1523. https://doi.org/10.1038/ s41467-019-09234-6.

18. Jiang M, Lash GE, Zeng S, Liu F, Han M, Long Y, et al. Differential expression of serum proteins before 20 weeks gestation in women with hypertensive disorders of pregnancy: a potential role for SH3BGRL3. Placenta. 2021;104: 20-30. https://doi.org/10.1016/j.placenta.2020.10.031.

19. Lynch AM, Murphy JR, Byers T, Gibbs RS, Neville MC, Giclas PC, et al. Alternative complement pathway activation fragment $\mathrm{Bb}$ in early pregnancy as a predictor of preeclampsia. Am J Obstetr Gynecol. 2008;198:385.e1-9.

20. Lynch A, Murphy J, Gibbs R, Levine R, Giclas P, Salmon J, et al. The interrelationship of complement-activation fragments and angiogenesisrelated factors in early pregnancy and their association with pre-eclampsia: complement, angiogenesis, pre-eclampsia and obesity. BJOG Int J Obstet Gynaecol. 2010;117:456-62. https://doi.org/10.1111/j.1471-0528.2009.02473.x.

21. Kim SM, Cho B-K, Kang MJ, Norwitz ER, Lee SM, Lee J, et al. Expression changes of proteins associated with the development of preeclampsia in maternal plasma: a case-control study. Proteomics. 2016;16:1581-9. https:// doi.org/10.1002/pmic.201500381.

22. Kolla V, Jenö P, Moes S, Lapaire O, Hoesli I, Hahn S. Quantitative proteomic (iTRAQ) analysis of 1st trimester maternal plasma samples in pregnancies at risk for preeclampsia. J Biomed Biotechnol. 2012;2012:305964.

23. Alrahmani L, Willrich MAV. The complement alternative pathway and preeclampsia. Curr Hypertens Rep. 2018;20:40. https://doi.org/10.1007/s11 906-018-0836-4.

24. Fakhouri F, Jablonski M, Lepercq J, Blouin J, Benachi A, Hourmant M, et al. Factor $\mathrm{H}$, membrane cofactor protein, and factor I mutations in patients with hemolysis, elevated liver enzymes, and low platelet count syndrome. Blood. 2008;1 12:4542-5. https://doi.org/10.1182/blood-2008-03-144691.
25. Salmon JE, Heuser C, Triebwasser M, Liszewski MK, Kavanagh D, Roumenina $L$, et al. Mutations in Complement Regulatory Proteins Predispose to Preeclampsia: A Genetic Analysis of the PROMISSE Cohort. M. Fisk N. PLoS Med. 2011:8:e1001013.

26. Burwick RM, Feinberg BB. Eculizumab for the treatment of preeclampsia/ HELLP syndrome. Placenta. 2013;34:201-3. https://doi.org/10.1016/j.pla centa.2012.11.014.

27. Csincsi Ál, Kopp A, Zöldi M, Bánlaki Z, Uzonyi B, Hebecker M, et al. Factor Hrelated protein 5 interacts with pentraxin 3 and the extracellular matrix and modulates complement activation. J Immunol. 2015;194:4963-73. https:// doi.org/10.4049/jimmunol.1403121.

28. Brocklebank V, Wood KM, Kavanagh D. Thrombotic Microangiopathy and the kidney. Clin J Am Soc Nephrol. 2018;13:300-17. https://doi.org/10.2215/ CJN.00620117

29. Chen Q, Manzke M, Hartmann A, Büttner M, Amann K, Pauly D, et al. Complement factor $\mathrm{H}$-related 5-hybrid proteins anchor Properdin and activate complement at self-surfaces. J Am Soc Nephrol. 2016;27:1413-25. https://doi.org/10.1681/ASN.2015020212.

30. Wilson MR, Zoubeidi A. Clusterin as a therapeutic target. Expert Opin Ther Targets. 2017;21:201-13. https://doi.org/10.1080/14728222.2017.1267142.

31. Mohanty G, Jena SR, Nayak J, Kar S, Samanta L. Quantitative proteomics decodes clusterin as a critical regulator of paternal factors responsible for impaired compensatory metabolic reprogramming in recurrent pregnancy loss. Andrologia. 2020;52:e13498.

32. Oztas E, Ozler S, Ersoy AO, Iskender CT, Sucak A, Ergin M, et al. Increased levels of serum clusterin is associated with intrauterine growth restriction and adverse pregnancy outcomes in preeclampsia. J Perinat Med. 2016;44. https://doi.org/10.1515/jpm-2015-0120.

33. Blumenstein M, McCowan LME, Wu S, Cooper GJS, North RA, SCOPE consortium. Plasma clusterin increased prior to small for gestational age (SGA) associated with preeclampsia and decreased prior to SGA in normotensive pregnancies. Reprod Sci. 2012;19:650-7.

34. Huppertz B. Traditional and new routes of Trophoblast invasion and their implications for pregnancy diseases. Int J Mol Sci. 2019;21. https://doi.org/1 0.3390/ijms21010289.

35. Mol BWJ, Roberts CT, Thangaratinam S, Magee LA, de Groot CJM, Hofmeyr GJ. Pre-eclampsia. Lancet. 2016;387(10022):999-1011. https://doi.org/10.101 6/S0140-6736(15)00070-7.

36. Jin R, Chen X, Han D, Luo X, Li H. Clusterin modulates transdifferentiation of non-small-cell lung cancer. BMC Cancer. 2017;17:661. https://doi.org/10.11 86/s12885-017-3649-y.

37. Jeong S, Ledee DR, Gordon GM, Itakura T, Patel N, Martin A, et al. Interaction of clusterin and matrix metalloproteinase-9 and its implication for epithelial homeostasis and inflammation. Am J Pathol. 2012;180:2028-39. https://doi.org/10.1016/j.ajpath.2012.01.025.

38. Geldenhuys J, Rossouw TM, Lombaard HA, Ehlers MM, Kock MM. Disruption in the regulation of immune responses in the placental subtype of preeclampsia. Front Immunol. 2018;9:1659. https://doi.org/10.3389/fimmu.2 018.01659.

39. Friedman SA, de Groot CJ, Taylor RN, Golditch BD, Roberts JM. Plasma cellular fibronectin as a measure of endothelial involvement in preeclampsia and intrauterine growth retardation. Am J Obstet Gynecol. 1994;170:838-41. https://doi.org/10.1016/S0002-9378(94)70295-0.

40. Wright SD, Craigmyle LS, Silverstein SC. Fibronectin and serum amyloid P component stimulate C3b- and C3bi-mediated phagocytosis in cultured human monocytes. J Exp Med. 1983;158:1338-43. https://doi.org/10.1084/ jem.158.4.1338.

41. Gelber SE, Brent E, Redecha P, Perino G, Tomlinson S, Davisson RL, et al. Prevention of defective placentation and pregnancy loss by blocking innate immune pathways in a syngeneic model of placental insufficiency. J Immunol. 2015:195:1129-38. https://doi.org/10.4049/jimmunol.1402220.

42. Moussavi-Harami SF, Annis DS, Ma W, Berry SM, Coughlin EE, Strotman LN, et al. Characterization of molecules binding to the 70K $\mathrm{N}$-terminal region of Fibronectin by IFAST purification coupled with mass spectrometry. J Proteome Res. 2013:12:3393-404. https://doi.org/10.1021/pr400225p.

\section{Publisher's Note}

Springer Nature remains neutral with regard to jurisdictional claims in published maps and institutional affiliations. 